\title{
Time-dependent Schrödinger perturbations of transition densities
}

\author{
by \\ KRZYSZTOF BogdAN (Wrocław), \\ Wolfhard Hansen (Bielefeld) and Tomasz Jakubowski (Wrocław)
}

This work is dedicated to Professor Jan Kisyński

\begin{abstract}
We construct the fundamental solution of $\partial_{t}-\Delta_{y}-q(t, y)$ for functions $q$ with a certain integral space-time relative smallness, in particular for those satisfying a relative Kato condition. The resulting transition density is comparable to the Gaussian kernel in finite time, and it is even asymptotically equal to the Gaussian kernel (in small time) under the relative Kato condition.

The result is generalized to arbitrary strictly positive and finite time-nonhomogeneous transition densities on measure spaces.

We also discuss specific applications to Schrödinger perturbations of the fractional Laplacian in view of the fact that the $3 \mathrm{P}$ Theorem holds for the fundamental solution corresponding to the operator.
\end{abstract}

1. Main results and overview. Let $d$ be a natural number. The Gaussian kernel on $\mathbb{R}^{d}$ is defined as

$$
g(s, x, t, y)=\frac{1}{(4 \pi(t-s))^{d / 2}} \exp \left(-\frac{|y-x|^{2}}{4(t-s)}\right) \quad \text { if }-\infty<s<t<\infty,
$$

and we let $g(s, x, t, y)=0$ if $s \geq t$. Here $x, y \in \mathbb{R}^{d}$ are arbitrary. It is well-known that $g$ is a time-homogeneous transition density with respect to the Lebesgue measure, $d z$, on $\mathbb{R}^{d}$. In particular, for $x, y \in \mathbb{R}^{d}$,

$$
\int_{\mathbb{R}^{d}} g(s, x, u, z) g(u, z, t, y) d z=g(s, x, t, y) \quad \text { if } s<u<t .
$$

2000 Mathematics Subject Classification: 47A55, 60J35, 60J57.

Key words and phrases: relative Kato condition, conditional process.

The first and third authors were partially supported by KBN. 
We will consider a Borel measurable function $q: \mathbb{R} \times \mathbb{R}^{d} \rightarrow \mathbb{R}$, and numbers $h>0$ and $0 \leq \eta<1$, such that for all $x, y \in \mathbb{R}^{d}$ and $s<t \leq s+h$,

$$
\int_{s}^{t} \int_{\mathbb{R}^{d}} \frac{g(s, x, u, z) g(u, z, t, y)}{g(s, x, t, y)}|q(u, z)| d z d u \leq \eta .
$$

THEOREM 1. There is a unique continuous transition density $\widetilde{g}$ such that

$$
\int_{s}^{\infty} \int_{\mathbb{R}^{d}} \widetilde{g}(s, x, t, y)\left[\partial_{t} \phi(t, y)+\Delta_{y} \phi(t, y)+q(t, y) \phi(t, y)\right] d y d t=-\phi(s, x),
$$

and

$$
\frac{\widetilde{g}(s, x, t, y)}{g(s, x, t, y)} \leq \frac{1}{1-\eta} \exp \left\{\frac{\eta}{(1-\eta) h}(t-s)\right\} .
$$

Here $s<t \in \mathbb{R}, x, y \in \mathbb{R}^{d}$, and $\phi \in C_{c}^{\infty}\left(\mathbb{R} \times \mathbb{R}^{d}\right)$ are arbitrary.

We consider $\Delta+q$ as an additive perturbation of the Laplacian $\Delta$ by the operator of multiplication by $q$ (i.e. a Schrödinger perturbation). According to $(2), \widetilde{g}$ is the integral kernel of a left inverse of $-\left(\partial_{t}+\Delta_{y}+q\right)$. Put differently, $f:(t, y) \mapsto \widetilde{g}(s, x, t, y)$ solves $\left(\partial_{t}-\Delta_{y}-q\right) f=\delta_{(s, x)}$ in the sense of distributions. Thus, $\widetilde{g}$ is a fundamental solution of $\partial_{t}-\Delta_{y}-q$ ([31]).

As we will see, $\widetilde{g}$ is constructed by means of $g$ and $q$ only, without referring to $\Delta$. A similar procedure applies to the fundamental solution of the fractional Laplacian $\Delta^{\alpha / 2}=-(-\Delta)^{\alpha / 2}$. At the end of the paper we give references and discuss these two important examples in some detail.

The primary goal of the paper is, however, to construct and estimate analogous time-dependent, or nonautonomous, Schrödinger perturbations for more general transition densities. We work under the appropriate assumption of relative smallness, or relative Kato condition on $q$. We give explicit upper bounds for the resulting transition density, which are new even in the autonomous Gaussian case. Our development is motivated by the role of the celebrated 3G Theorem in studying Schrödinger-type perturbations of Green functions [19, 20, 21] (see also [12], [11], [9], [3], [4], [10]). Another motivation comes from a recent estimate, the $3 \mathrm{P}$ Theorem of [5] for the fundamental solution of $\Delta^{\alpha / 2}$. The estimate was used in [5] to construct the transition density of autonomous gradient perturbations of $\Delta^{\alpha / 2}$, in a way resembling the above mentioned study of Schrödinger perturbations of Green functions by means of the 3G Theorem (see also [23]). [5] and the present paper show that a perturbation technique similar to that of [20] applies even more naturally to the parabolic Green function (that is, the fundamental solution, or transition density). We propose an explicit construction of the perturbed transition density under a minimum of assumptions, corresponding to the generality of $[19,20,21,10]$. We refer the reader to [13, Theorem 3.5, p. 71], 
[24], and [14, Theorem 19, Ch.VIII] for a selection of results in the perturbation theory of linear operators and semigroups on Banach spaces. For recent developments in Schrödinger perturbations of time-nonhomogeneous transition probabilities we refer the reader to [17], [27], [16]. We point out that our main estimate, Theorem 3 below, is more precise and explicit than those mentioned above. Accordingly, it also strengthens in the present context the celebrated Khas'minski's lemma, one of the main tools in the probabilistic theory of Schrödinger perturbations of generators of Markov processes (see, e.g., [11], [16]). This strengthening is of independent interest - the estimate is valid in the full range of times, rather than only in small time intervals, and the proof gives a deeper insight into the interplay between individual terms of the series involved.

To explain the connection of Theorem 1 to our general results we note that $\widetilde{g}$ satisfies the following equation for all $x, y \in \mathbb{R}^{d}$ and $s, t \in \mathbb{R}$ :

$$
\widetilde{g}(s, x, t, y)=g(s, x, t, y)+\iint_{\mathbb{R} X} g(s, x, u, z) q(u, z) \widetilde{g}(u, z, t, y) d z d u
$$

(see the proof of Theorem 2). This is called Duhamel's formula or perturbation formula. The equation implicitly defines the perturbed transition density in this situation (and in more general ones), which we will now discuss.

Consider an arbitrary set $X$ with a $\sigma$-algebra $\mathcal{M}$ and a (nonnegative) measure $m$ defined on $\mathcal{M}$. To simplify the notation we will write $d z$ for $m(d z)$ in what follows. Consider the $\sigma$-algebra $\mathcal{B}$ of Borel subsets of $\mathbb{R}$, and the Lebesgue measure, $d u$, defined on $\mathcal{B}$. The space-time, $\mathbb{R} \times X$, will be equipped with the $\sigma$-algebra $\mathcal{B} \times \mathcal{M}$ and product measure $d u d z=d u m(d z)$.

Let $p$ be a $\mathcal{B} \times \mathcal{M} \times \mathcal{B} \times \mathcal{M}$-measurable function defined (everywhere) on $\mathbb{R} \times X \times \mathbb{R} \times X$. We will call $p$ a transition density on $X$ if

$$
\begin{aligned}
p(s, x, t, y)=0 & \text { whenever } s \geq t, \\
0<p(s, x, t, y)<\infty & \text { when } s<t, x, y \in X,
\end{aligned}
$$

and the following Chapman-Kolmogorov equation holds: if $s<u<t$ then

$$
\int_{X} p(s, x, u, z) p(u, z, t, y) d z=p(s, x, t, y), \quad x, y \in X .
$$

Note that we require strict positivity in (4), while (3) is merely a convention. By (4) and (5), $m$ is necessarily $\sigma$-finite on $X$. The reader may regard $s$ and $x$ in $p(s, x, t, y)$ as the starting time and position of a variable mass spreading in $X$, and $t, y$ as the ending time and position. Thus, $\int_{X} p(s, x, t, y) d y$ is the total mass at time $t$. We will say that transition densities $p^{\prime}$ and $p^{\prime \prime}$ on $X$ are comparable locally in time if for every $h>0$ there is a (finite) constant $c=c(h)$ such that $c^{-1} p^{\prime \prime}(s, x, t, y) \leq p^{\prime}(s, x, t, y) \leq c p^{\prime \prime}(s, x, t, y)$ for all $x, y \in X$ provided $s<t<s+h$. We will say that they are asymptotically equal if $c(h)$ may be chosen in such a way that $c(h) \rightarrow 1$ as $h \rightarrow 0^{+}$. 
All the functions discussed below will be assumed measurable with respect to the relevant $\sigma$-algebras, usually with respect to $\mathcal{B} \times \mathcal{M}$.

If $q$ is a nonnegative function defined on $\mathbb{R} \times \mathcal{M}$, then we let

$$
\eta^{*}(q)=\inf \eta,
$$

where the infimum is taken over all $\eta>0$ with the property that there exists $h>0$ such that for all $x, y \in \mathbb{R}^{d}$ and $s<t \leq s+h$,

$$
\int_{s}^{t} \int_{X} p(s, x, u, z) q(u, z) p(u, z, t, y) d z d u \leq \eta p(s, x, t, y) .
$$

We will say that a (signed) function $q: \mathbb{R} \times X \rightarrow \mathbb{R}$ is relatively bounded (at small times, with respect to $p$ and $m$ ) if $0 \leq \eta^{*}(|q|)<\infty$. We will say that $q$ is relatively small if $0 \leq \eta^{*}(|q|)<1$, and we will say that $q$ is relatively Kato $\left.{ }^{1}\right)$ if $\eta^{*}(|q|)=0$.

TheOREM 2. If $q: \mathbb{R} \times X \rightarrow \mathbb{R}$ is relatively small, then there is a unique transition density $\widetilde{p}$ on $X$ locally in time comparable with $p$, such that for all $s, t \in \mathbb{R}$ and $x, y \in X$ we have

$$
\widetilde{p}(s, x, t, y)=p(s, x, t, y)+\int_{\mathbb{R} X} p(s, x, u, z) q(u, z) \widetilde{p}(u, z, t, y) d z d u .
$$

If $q$ is relatively Kato, then $\widetilde{p}$ and $p$ are asymptotically equal.

We note that explicit upper and lower bounds for $\widetilde{p}$ exist expressed in terms of $\eta^{*}\left(q_{+}\right)$and $\eta^{*}\left(q_{-}\right)$(see Theorem 3 and (27), and also (41)).

The paper is organized as follows. In Section 2 we describe the basic formalism of the perturbation series in the context of the Chapman-Kolmogorov equation. In Section 3 we reformulate the relative boundedness and smallness of $q$. By a combinatorial argument, we prove our main estimate, Theorem 3, for the perturbation series for relatively small $q \geq 0$. In Section 4 we consider signed relatively small $q$ and we prove Theorem 2. In Section 5 we discuss in more detail Schrödinger perturbations of the transition densities of Laplacian and fractional Laplacian, and we give the proof of Theorem 1. In view of the fact that the transition density of the fractional Laplacian (but not that of the Laplacian) satisfies the 3P Theorem, in Section 5 we characterize the relative Kato condition by means of the parabolic Kato condition studied in [30].

Our main goal is to give applications motivating the use of relative smallness in perturbation theory of transition densities, along with a self-contained exposition of some of the relevant techniques. We do not aim at full generality here. Possible and forthcoming generalizations are mentioned in Section 6, where we also give a probabilistic interpretation of our results.

$\left({ }^{1}\right)$ A different "relative Kato condition" is used in [7, (4)] and [8, (2)]. 
2. Algebra of perturbation series. Let $q: \mathbb{R} \times X \rightarrow \mathbb{R}$. The identities we intend to prove below rely merely on changing the order of integration, which is justified if the integrals involved are absolutely convergent or nonnegative. We shall first consider the latter situation and we will assume that $q \geq 0$.

Duhamel's formula (8) suggests the following definitions. For $s, t \in \mathbb{R}$ and $x, y \in X$, we let $p_{0}(s, x, t, y)=p(s, x, t, y)$ and

$$
p_{n}(s, x, t, y)=\int_{s}^{t} \int_{X} p_{n-1}(s, x, u, z) p(u, z, t, y) q(u, z) d z d u
$$

for $n \geq 1$, and we define the perturbation of $p$ by $q$,

$$
\widetilde{p}_{q}(s, x, t, y)=\sum_{n=0}^{\infty} p_{n}(s, x, t, y), \quad x, y \in X, s, t \in \mathbb{R} .
$$

If $s \geq t$, then $p_{n}(s, x, t, y)=0$ for every $n \geq 0$ and hence $\widetilde{p}_{q}(s, x, t, y)=0$.

Since $p(s, x, t, y)=0$ for $s \geq t$, we could write (9) as

$$
p_{n}(s, x, t, y)=\int_{\mathbb{R} X} p_{n-1}(s, x, u, z) p(u, z, t, y) q(u, z) d z d u
$$

for all $s, t \in \mathbb{R}$ and $x, y \in X$, so the reader should not be alarmed if we occasionally simplify our notation in this way.

Lemma 1. For all $s<u<t, x, y \in X$, and $n=0,1, \ldots$,

$$
\sum_{m=0}^{n} \int_{X} p_{m}(s, x, u, z) p_{n-m}(u, z, t, y) d z=p_{n}(s, x, t, y) .
$$

Proof. We note that (11) is true for $n=0$ by (5). Assume that $n \geq 1$ and (11) holds for $n-1$. The sum of the first $n$ terms in (11) can be dealt with by induction:

$$
\begin{aligned}
& \sum_{m=0}^{n-1} \int_{X} p_{m}(s, x, u, z) p_{n-m}(u, z, t, y) d z \\
= & \sum_{m=0}^{n-1} \int_{X} p_{m}(s, x, u, z) \int_{u}^{t} \int_{X} p_{n-1-m}(u, z, r, w) p(r, w, t, y) q(r, w) d w d r d z \\
= & \int_{u}^{t} \int_{X}\left(\sum_{m=0}^{n-1} \int_{X} p_{m}(s, x, u, z) p_{n-1-m}(u, z, r, w) d z\right) p(r, w, t, y) q(r, w) d w d r \\
= & \int_{u}^{t} \int_{X} p_{n-1}(s, x, r, w) p(r, w, t, y) q(r, w) d w d r .
\end{aligned}
$$


By (9), the $(n+1)$-st term is

$$
\begin{aligned}
& \int_{X} p_{n}(s, x, u, z) p_{0}(u, z, t, y) d z \\
& \quad=\int_{X}^{u} \int_{s} \int_{X} p_{n-1}(s, x, r, w) p(r, w, u, z) q(r, w) d w d r p(u, z, t, y) d z \\
& =\int_{s}^{u} \int_{X} p_{n-1}(s, x, r, w) p(r, w, t, y) q(r, w) d w d r .
\end{aligned}
$$

and (11) follows on adding (12) and (13).

We next prove the Chapman-Kolmogorov equation for $\widetilde{p}_{q}=\sum_{n=0}^{\infty} p_{n}$.

Lemma 2. For all $s<u<t$ and $x, y \in X$,

$$
\int_{X} \widetilde{p}_{q}(s, x, u, z) \widetilde{p}_{q}(u, z, t, y) d z=\widetilde{p}_{q}(s, x, t, y) .
$$

Proof. By Lemma 1,

$$
\begin{aligned}
\int_{X} \widetilde{p}_{q}(s, x, u, z) \widetilde{p}_{q}(u, z, t, y) d z & =\int_{X} \sum_{i=0}^{\infty} p_{i}(s, x, u, z) \sum_{j=0}^{\infty} p_{j}(u, z, t, y) d z \\
& =\sum_{n=0}^{\infty} \sum_{m=0}^{n} \int_{X} p_{m}(s, x, u, z) p_{n-m}(u, z, t, y) d z \\
& =\sum_{n=0}^{\infty} p_{n}(s, x, t, y)=\widetilde{p}_{q}(s, x, t, y) .
\end{aligned}
$$

We will need the following extension of (9).

Lemma 3. For all $n=1,2, \ldots, m=0,1, \ldots, n-1, s, t \in \mathbb{R}$, and $x, y \in X$,

$$
p_{n}(s, x, t, y)=\int_{s}^{t} \int_{X} p_{n-1-m}(s, x, u, z) p_{m}(u, z, t, y) q(u, z) d z d u .
$$

Proof. For $m=0$, equality (14) holds by definition of $p_{n}$. In particular, this proves our claim for $n=1$. If the claim is proved for some $n \geq 1$, then, for every $m=1, \ldots, n$,

$$
\begin{aligned}
p_{n+1}(s, x, t, y) & =\int_{\mathbb{R}} \int_{X} p_{n}(s, x, u, z) p(u, z, t, y) q(u, z) d z d u \\
= & \int_{\mathbb{R} X} \int_{\mathbb{R} X} \int_{X} p_{n-1-(m-1)}(s, x, w, v) p_{m-1}(w, v, u, z) q(v, w) d w d v \\
& \quad \times p(u, z, t, y) q(u, z) d z d u \\
& =\int_{\mathbb{R} X} \int_{n-m} p_{n-m}(s, x, w, v) p_{m}(w, v, t, y) q(v, w) d w d v .
\end{aligned}
$$


3. Estimate from above. In this section we will only consider relatively bounded $q \geq 0$. Given $s<t$, we let $I(s, t)$ be the smallest number such that for all $x, y \in X$,

$$
\int_{s}^{t} \int_{X} p(s, x, u, z) p(u, z, t, y) q(u, z) d z d u \leq I(s, t) p(s, x, t, y) .
$$

Relative boundedness of $q$ implies that $I(s, t)$ is finite if $t-s$ is small. The following lemma implies that $I(s, t)$ is then finite for all $s<t$.

Lemma 4. $I(s, v) \leq I(s, t)+I(t, v)$ whenever $s<t<v$.

Proof. Let $s<t<v$ and $x, y \in X$. We have

$$
\begin{aligned}
& \int_{s}^{v} \int_{X} p(s, x, u, z) p(u, z, v, y) q(u, z) d z d u=\int_{s}^{t}+\int_{t}^{v} \\
&=\int_{s}^{t} \int_{X} \int_{X} p(s, x, u, z) p(u, z, t, w) p(t, w, v, y) d w q(u, z) d z d u \\
& \quad+\int_{t}^{v} \int_{X} \int_{X} p(s, x, t, w) p(t, w, u, z) p(u, z, v, y) d w q(u, z) d z d u \\
& \leq {[I(s, t)+I(t, v)] \int_{X} p(s, x, t, w) p(t, w, v, y) d w } \\
&= {[I(s, t)+I(t, v)] p(s, x, v, y) . }
\end{aligned}
$$

This subadditivity and the relative boundedness yield the following.

Lemma 5. If $\eta>\eta^{*}(q)$, then there is $\beta \geq 0$ such that

$$
\int_{s}^{t} \int_{X} p(s, x, u, z) p(u, z, t, y) q(u, z) d z d u \leq[\eta+\beta(t-s)] p(s, x, t, y)
$$

whenever $s<t \in \mathbb{R}$ and $x, y \in X$.

Proof. For $\eta>\eta^{*}(q)$ let $h>0$ be as in the line preceding (7). If $k$ is a natural number and $s+(k-1) h<t \leq s+k h$, then $k<1+(t-s) / h$, and, by Lemma $4, I(s, t) \leq k \eta \leq \eta+\eta(t-s) / h$. We can take

$$
\beta=\eta / h \text {. }
$$

Conversely, if (16) holds with some finite $\eta$ and $\beta$, then $q$ is relatively bounded, and $\eta^{*}(q) \leq \eta$. Also, (16) with $0 \leq \eta<1$ (and some finite $\beta$ ) characterizes relative smallness, and (16) being true for every $\eta \geq 0$ (with some finite $\beta$ ) is equivalent to the relative Kato condition. Thus, our focus in (16) is on the value of $\eta$; the term $\beta(t-s)$ is merely a technically convenient replacement of $h$. 
In the remainder of the section we will assume that (16) holds with $0 \leq \eta<1$ and (finite) $\beta \geq 0$. For instance, every bounded (nonnegative) $q$ satisfies that assumption with $\eta=0$ and $\beta=\sup _{\mathbb{R} \times X} q(u, z)$. Indeed,

$$
\int_{s}^{t} \int_{X} p(s, x, u, z) p(u, z, t, y) q(u, z) d z d u \leq[\sup q](t-s) p(s, x, t, y) .
$$

We shall need the following identity.

Lemma 6. For all $n=0,1, \ldots$ and $\xi, \nu, \eta \in \mathbb{R}$,

$$
\begin{aligned}
\sum_{m=0}^{n} \sum_{k=0}^{n-m} \sum_{j=0}^{m}\left(\begin{array}{c}
n-m \\
k
\end{array}\right)\left(\begin{array}{c}
m \\
j
\end{array}\right) \frac{\xi^{k} \nu^{j}}{k ! j !} \eta^{n-k-j} & =\sum_{r=0}^{n}\left(\begin{array}{c}
n+1 \\
r+1
\end{array}\right) \frac{(\xi+\nu)^{r}}{r !} \eta^{n-r} .
\end{aligned}
$$

Proof. Let $j, r$ be integers with $0 \leq j \leq r \leq n$. There are $\left(\begin{array}{l}n+1 \\ r+1\end{array}\right)$ subsets of $\{0,1, \ldots, n\}$ having $r+1$ elements. Such a subset, $\left\{i_{1}, \ldots, i_{r+1}\right\}$ with $i_{1}<$ $\cdots<i_{r+1}$, may be chosen by first fixing $m:=i_{j+1} \in\{j, j+1, \ldots, n-r+j\}$ and then taking $j$ integers $0 \leq i_{1}<\cdots<i_{j}<m$, and $r-j$ integers $m<i_{j+2}<\cdots<i_{r+1} \leq n$. This shows that (for every such $j$ )

$$
\sum_{j \leq m \leq n-r+j}\left(\begin{array}{c}
m \\
j
\end{array}\right)\left(\begin{array}{c}
n-m \\
r-j
\end{array}\right)=\left(\begin{array}{c}
n+1 \\
r+1
\end{array}\right)
$$

(cf. $[15,(5.26)])$. Considering $k=r-j$ we see that the conditions $0 \leq r \leq n$, $0 \leq j \leq r, j \leq m \leq n-r+j$ are equivalent to $0 \leq m \leq n, 0 \leq k \leq n-m$, $0 \leq j \leq m$. Therefore

$$
\begin{aligned}
\sum_{m=0}^{n} \sum_{k=0}^{n-m} \sum_{j=0}^{m}\left(\begin{array}{c}
n-m \\
k
\end{array}\right) & \left(\begin{array}{c}
m \\
j
\end{array}\right) \frac{\xi^{k} \nu^{j}}{k ! j !} \eta^{n-k-j} \\
& =\sum_{r=0}^{n} \sum_{j=0}^{r} \sum_{m=j}^{n+j-r}\left(\begin{array}{c}
m \\
j
\end{array}\right)\left(\begin{array}{c}
n-m \\
r-j
\end{array}\right) \frac{\nu^{j} \xi^{r-j}}{j !(r-j) !} \eta^{n-r} \\
& =\sum_{r=0}^{n}\left(\begin{array}{c}
n+1 \\
r+1
\end{array}\right) \frac{(\nu+\xi)^{r}}{r !} \eta^{n-r} .
\end{aligned}
$$

The following result is our main technical observation, justifying the assumption (16).

Lemma 7. For all $n=0,1,2, \ldots, x, y \in X$, and $s, t \in \mathbb{R}$,

$$
p_{n}(s, x, t, y) \leq p(s, x, t, y) \sum_{k=0}^{n}\left(\begin{array}{l}
n \\
k
\end{array}\right) \frac{(\beta(t-s))^{k}}{k !} \eta^{n-k} .
$$


Proof. Of course, (19) holds for $n=0$. By Lemma 3, induction, Lemma 6, and (16),

$$
\begin{aligned}
(n+1) & p_{n+1}(s, x, t, y) \\
= & \sum_{m=0}^{n} \int_{s}^{t} \int_{X} p_{n-m}(s, x, u, z) p_{m}(u, z, t, y) q(u, z) d z d u \\
\leq & \int_{s}^{t} \int_{X} p(s, x, u, z) p(u, z, t, y) q(u, z) d z d u \\
& \times \sum_{m=0}^{n} \sum_{k=0}^{n-m} \sum_{j=0}^{m}\left(\begin{array}{c}
n-m \\
k
\end{array}\right)\left(\begin{array}{c}
m \\
j
\end{array}\right) \frac{(\beta(u-s))^{k}}{k !} \eta^{n-m-k} \frac{(\beta(t-u))^{j}}{j !} \eta^{m-j} \\
= & \int_{s}^{t} \int_{X} p(s, x, u, z) p(u, z, t, y) q(u, z) d z d u \sum_{r=0}^{n}\left(\begin{array}{c}
n+1 \\
r+1
\end{array}\right) \frac{(\beta(t-s))^{r}}{r !} \eta^{n-r} \\
\leq & p(s, x, t, y)[\eta+\beta(t-s)] \sum_{r=0}^{n}\left(\begin{array}{c}
n+1 \\
r+1
\end{array}\right) \frac{(\beta(t-s))^{r}}{r !} \eta^{n-r} \\
= & p(s, x, t, y)\left[\sum_{r=0}^{n}\left(\begin{array}{c}
n+1 \\
r
\end{array}\right) \frac{n+1-r}{r+1} \frac{(\beta(t-s))^{r}}{r !} \eta^{n+1-r}\right. \\
\leq & \left.\left.(n+1) p(s, x, t, y) \sum_{r=0}^{n+1}\left(\begin{array}{c}
n+1 \\
r
\end{array}\right) \frac{(\beta(t-s))^{r}}{r !} \eta^{n+1-r} . \text { - } \begin{array}{c}
n+1 \\
r
\end{array}\right) r \frac{(\beta(t-s))^{r}}{r !} \eta^{n+1-r}\right]
\end{aligned}
$$

THEOREM 3. If $q$ satisfies (16) with $\eta<1$, then, for all $s<t$ and $x, y \in X$,

$$
\widetilde{p}_{q}(s, x, t, y) \leq \frac{1}{1-\eta} \exp \left(\frac{\beta}{1-\eta}(t-s)\right) p(s, x, t, y) .
$$

Proof. By (10) and Lemma 7,

$$
\widetilde{p}_{q}(s, x, t, y) \leq p(s, x, t, y) \sum_{k=0}^{\infty} \frac{(\beta(t-s))^{k}}{k !} \sum_{n=k}^{\infty}\left(\begin{array}{l}
n \\
k
\end{array}\right) \eta^{n-k},
$$

where

$$
\begin{aligned}
\sum_{n=k}^{\infty}\left(\begin{array}{l}
n \\
k
\end{array}\right) \eta^{n-k} & =\frac{1}{k !} \frac{d^{k}}{d \eta^{k}} \sum_{n=0}^{\infty} \eta^{n}=\frac{1}{k !} \frac{d^{k}}{d \eta^{k}} \frac{1}{1-\eta}=\frac{1}{k !} \frac{k !}{(1-\eta)^{k+1}} \\
& =\frac{1}{(1-\eta)^{k+1}} .
\end{aligned}
$$


Therefore

$$
\begin{aligned}
\widetilde{p}_{q}(s, x, t, y) & \leq p(s, x, t, y) \frac{1}{1-\eta} \sum_{k=0}^{\infty} \frac{(\beta(t-s) /(1-\eta))^{k}}{k !} \\
& =\frac{1}{1-\eta} \exp \left(\frac{\beta}{1-\eta}(t-s)\right) p(s, x, t, y) . \mathbf{-}
\end{aligned}
$$

4. Small signed perturbations. We will present some immediate consequences of Theorem 3 for signed $q$. Let $q_{+}=\max (q, 0)$ and $q_{-}=$ $\max (-q, 0)$, so that $q=q_{+}-q_{-}$. It will be convenient to consider the following integral kernels over space-time $\mathbb{R} \times X$ :

$$
\begin{aligned}
\operatorname{Pf}(s, x) & :=P_{p} f(s, x):=\int_{\mathbb{R} X} \int_{X} p(s, x, u, z) f(u, z) d z d u, \\
P^{q} f(s, x) & :=P_{p}^{q} f(s, x):=\int_{\mathbb{R} X} p(s, x, u, z) f(u, z) q(u, z) d z d u .
\end{aligned}
$$

For $t \in \mathbb{R}$ and $y \in X$ we have

$$
\begin{aligned}
\left(P^{q} p(\cdot, \cdot, t, y)\right)(s, x) & =\int_{\mathbb{R} X} p(s, x, u, z) p(u, z, t, y) q(u, z) d z d u \\
& =p_{1}(s, x, t, y), \quad s \in \mathbb{R}, x \in X .
\end{aligned}
$$

Using Lemma 3 we find by induction that, for every natural $n$,

$$
\left(\left(P^{q}\right)^{n} p(\cdot, \cdot, t, y)\right)(s, x)=P^{q}\left(p_{n-1}(\cdot, \cdot, t, y)\right)(s, x)=p_{n}(s, x, t, y) .
$$

For ease of notation, we can write $p_{n}(s, x, t, y)=\left(P^{q}\right)^{n} p(s, x, t, y)$, or even $p_{n}=\left(P^{q}\right)^{n} p$. In view of (10) we define (for signed $q$ )

$$
\widetilde{p}_{q}=\sum_{n=0}^{\infty}\left(P^{q}\right)^{n} p,
$$

whenever the integrals and the sum are nonnegative or absolutely convergent. Given (signed) $q_{1}$ and $q_{2}$ we consider the effect of two consecutive perturbations,

$$
\widetilde{\left(\widetilde{p}_{q_{1}}\right)_{q_{2}}}:=\sum_{n=0}^{\infty}\left(P_{\widetilde{p}_{q_{1}}}^{q_{2}}\right)^{n} \widetilde{p}_{q_{1}} .
$$

Here $P_{\widetilde{p}_{q_{1}}}^{q_{2}} f(s, x)=\int_{\mathbb{R}} \int_{X} \widetilde{p}_{q_{1}}(s, x, u, z) f(u, z) q_{2}(u, z) d z d u$ (cf. (22)). The following is a special case of a general result on perturbations of integral kernels (see, e.g., [28, Problem 1.13]).

LEMMA 8. Under the assumptions of nonnegativity or absolute convergence,

$$
\widetilde{\left(\widetilde{p}_{q_{1}}\right)_{q_{2}}}=\widetilde{p}_{q_{1}+q_{2}}
$$


Proof. It is not hard to verify that

$$
P_{\widetilde{p}_{q_{1}}}^{q_{2}}=\sum_{k=0}^{\infty}\left(P^{q_{1}}\right)^{k} P^{q_{2}} .
$$

It follows that

$$
\begin{aligned}
\widetilde{\left(\widetilde{p}_{q_{1}}\right)_{q_{2}}} & =\sum_{n=0}^{\infty}\left(P_{\widetilde{p}_{q_{1}}}^{q_{2}}\right)^{n} \widetilde{p}_{q_{1}}=\sum_{n=0}^{\infty}\left(\sum_{k=0}^{\infty}\left(P^{q_{1}}\right)^{k} P^{q_{2}}\right)^{n} \sum_{l=0}^{\infty}\left(P^{q_{1}}\right)^{l} p \\
& =\sum_{n=0}^{\infty} \sum_{\sigma \in\{1,2\}^{n}} P^{q_{\sigma(1)}} \ldots P^{q_{\sigma(n)}} p=\widetilde{p}_{q_{1}+q_{2}} .
\end{aligned}
$$

Proof of Theorem 2. We assume that $\eta^{*}(|q|)<1$, in particular $\eta^{*}\left(q_{-}\right)<1$ and $\eta^{*}\left(q_{+}\right)<1$. By Theorem 3, $\widetilde{p}_{q_{-}}=\sum_{n=0}^{\infty}\left(P^{q_{-}}\right)^{n} p$ is convergent, hence

$$
\widetilde{p}_{-q_{-}}=\sum_{n=0}^{\infty}(-1)^{n}\left(P^{q_{-}}\right)^{n} p
$$

is well defined and $\left|\widetilde{p}_{-q_{-}}\right| \leq \widetilde{p}_{q_{-}}$. Therefore the arguments of Section 2 apply, in particular $\widetilde{p}_{-q_{-}}$satisfies the Chapman-Kolmogorov equation (see Lemma 2). We will prove that $\widetilde{p}_{-q_{-}} \geq 0$. Indeed, let $\eta^{*}\left(q_{-}\right)<\eta<1$ and let $h>0$ be as required between (6) and (7), that is, for all $s<t \leq s+h$,

$$
P^{q_{-}} p(s, x, t, y) \leq \eta p(s, x, t, y) \leq p(s, x, t, y), \quad x, y \in X .
$$

Then $\left(P^{q_{-}}\right)^{n+1} p(s, x, t, y) \leq \eta\left(P^{q_{-}}\right)^{n} p(s, x, t, y)$ for $n=1,2, \ldots$, and hence

$$
\widetilde{p}_{-q_{-}}=\left(p-P^{q_{-}} p\right)+\left(\left(P^{q_{-}}\right)^{2} p-\left(P^{q_{-}}\right)^{3} p\right)+\cdots \geq(1-\eta) p .
$$

In particular, $\widetilde{p}_{-q_{-}}(s, x, t, y) \geq 0$ provided $s<t<s+h$. By the ChapmanKolmogorov equation, $\widetilde{p}_{-q_{-}}(s, x, t, y)$ is nonnegative for all times $s, t$. Also,

$$
\widetilde{p}_{-q_{-}}=p-\left(P^{q_{-}} p-\left(P^{q_{-}}\right)^{2} p\right)-\left(\left(P^{q_{-}}\right)^{3} p-\left(P^{q_{-}}\right)^{4} p\right)+\cdots \leq p
$$

provided $s<t<s+h$. Therefore,

$$
\widetilde{p}_{-q_{-}}(s, x, t, y) \leq p(s, x, t, y) \quad \text { for all } s, t \in \mathbb{R}, x, y \in X .
$$

This further shows that the perturbation of $\widetilde{p}_{-q_{-}}$by $q_{+}$is well defined, and $\widetilde{\left(\widetilde{p}_{-q_{-}}\right)_{q_{+}}} \leq \widetilde{p}_{q_{+}}$(cf. (9)). On the other hand, the (nonnegative) series defining $\widetilde{p}_{|q|}$ is convergent. Thus, by Lemma 8 and the considerations above,

$$
\widetilde{p}_{-q_{-}} \leq \widetilde{\left(\widetilde{p}_{-q_{-}}\right)_{q_{+}}}=\widetilde{p}_{q} \leq \widetilde{p}_{q_{+}} .
$$

We note that if $n$ is a natural number and $s<t<s+n h$, then ChapmanKolmogorov, (25), Theorem 3, and (17) imply that, for all $x, y \in X$,

$$
(1-\eta)^{n} \leq \frac{\widetilde{p}_{q}(s, x, t, y)}{p(s, x, t, y)} \leq \frac{1}{1-\eta} \exp \frac{n \eta}{1-\eta} .
$$


By Theorem 3 (applied to $|q|$ ), the series $\sum_{n=0}^{\infty}\left(P^{q}\right)^{n} p$ is absolutely convergent and hence

$$
\left(I-P^{q}\right) \widetilde{p}_{q}=\sum_{n=0}^{\infty}\left(P^{q}\right)^{n} p-\sum_{n=0}^{\infty}\left(P^{q}\right)^{n+1} p=p .
$$

Thus $\widetilde{p}=\widetilde{p}_{q}$ solves $(8)$.

To prove the uniqueness of the solution, let $\widetilde{p}$ be any transition density which locally in time is comparable with $p$. Then the integral in (8) is absolutely convergent. This follows from Lemma 5 (note that the domain of integration in (8) is merely $(s, t) \times X)$. Therefore $\left(I-P^{q}\right) \widetilde{p}=p$ and

$$
\widetilde{p}=\sum_{n=0}^{\infty}\left(P^{q}\right)^{n}\left(I-P^{q}\right) \widetilde{p}=\sum_{n=0}^{\infty}\left(P^{q}\right)^{n} p=\widetilde{p}_{q} .
$$

If $\eta^{*}(|q|)=0$ then we can have $0<\eta<1$ arbitrarily small in the above discussion, therefore $\widetilde{p}_{q}$ and $p$ are asymptotically equal by (27).

Let $\widetilde{P}=P_{\widetilde{p}}$, that is, $\widetilde{P} f(s, x)=\int_{\mathbb{R}} \int_{X} \widetilde{p}(s, x, u, z) f(u, z) d z d u$, where $\widetilde{p}=\widetilde{p}_{q}$. Note that

$$
\widetilde{P}=\sum_{n=0}^{\infty}\left(P^{q}\right)^{n} P .
$$

Following [30], [16], we will say that $q: \mathbb{R} \times X \rightarrow \mathbb{R}$ belongs to the parabolic (space-time) Kato class for $p$ if

$$
\lim _{h \rightarrow 0+} \sup _{s \in \mathbb{R}, x \in X} \int_{X}^{s+h} \int_{s}^{s+h} p(s, x, u, z)|q(u, z)| d u d z=0
$$

and

$$
\lim _{h \rightarrow 0+} \sup _{t \in \mathbb{R}, y \in X} \int_{X} \int_{t-h}^{t} p(u, z, t, y)|q(u, z)| d u d z=0 .
$$

We say that $p$ is probabilistic if

$$
\int_{X} p(s, x, t, y) d y=1 \quad \text { for } s<t, x \in X .
$$

Lemma 9. If $p$ is probabilistic, $p(s, x, t, y)=p(s, y, t, x)$ for $s, t \in \mathbb{R}$, $x, y \in X$, and $q$ is relatively Kato for $p$, then $q$ is in the parabolic Kato class.

Proof. Let $h, \eta \geq 0$, and assume that for all $x, y \in \mathbb{R}^{d}$ and $s<t \leq s+h$,

$$
\int_{s}^{t} \int_{X} p(s, x, u, z) p(u, z, t, y)|q(u, z)| d z d u \leq \eta p(s, x, t, y) .
$$


Integrating this with respect to $d y$ we obtain

$$
\int_{s}^{t} \int_{X} p(s, x, u, z)|q(u, z)| d z d u \leq \eta .
$$

Thus, $\eta^{*}(|q|)=0$ yields (29). Integrating with respect to $d x$ instead, we obtain (30).

We note that Corollary 11 below gives a special converse to Lemma 9 .

In view of Theorem 3, the relative Kato condition seems more intrinsic to Schrödinger perturbations than the parabolic Kato condition (see [16, Lemma 5.2] and [27, Theorem 3.10]), but the former may be cumbersome to verify in specific situations. For instance, the relative Kato condition for the Gaussian kernel is rather difficult to explicitly characterize (as opposed to that for the transition density of $\Delta^{\alpha / 2}, 0<\alpha<2$, see below). It should be noted that each transition density $p$ determines a specific class of relatively Kato functions $q$, and a detailed analysis is required to exhibit interesting (unbounded) $q$. We also remark that the relative Kato condition may be interpreted as a Kato condition for bridges (see Section 6).

5. Examples. In this section we assume that $X=\mathbb{R}^{d}, d \geq 1, \mathcal{M}$ is the $\sigma$-algebra of Borel subsets of $\mathbb{R}^{d}$, and $d z$ is the Lebesgue measure on $\mathbb{R}^{d}$. Let $\alpha \in(0,2)$. Our aim is to discuss the transition density, $\widetilde{p}(s, x, t, y)$, of $\Delta^{\alpha / 2}+q$, where $q$ is relatively small. To state our estimates of $\widetilde{p}$, we specialize to

$$
p(s, x, t, y)=p_{t-s}(y-x),
$$

where $x, y \in \mathbb{R}^{d}, s<t$, and $p_{t}$ is the smooth real-valued function on $\mathbb{R}^{d}$ determined by

$$
\int_{\mathbb{R}^{d}} p_{t}(z) e^{i z \cdot \xi} d z=e^{-t|\xi|^{\alpha}}, \quad \xi \in \mathbb{R}^{d}, t>0 .
$$

In particular, for $\alpha=1$ we have

$$
p_{t}(z)=\Gamma((d+1) / 2) \pi^{-(d+1) / 2} \frac{t}{\left(|z|^{2}+t^{2}\right)^{(d+1) / 2}},
$$

the Cauchy convolution semigroup. Note that for every $\alpha \in(0,2)$,

$$
p_{t}(z)=t^{-d / \alpha} p_{1}\left(t^{-1 / \alpha} z\right), \quad t>0, z \in \mathbb{R}^{d} .
$$

This follows from (33). We let $p(s, x, t, y)=0$ if $s \geq t$. By the definitions, $p$ is time- and space-homogeneous: for all $s, t, h \in \mathbb{R}$ and $x, y, z \in \mathbb{R}^{d}$ we have

$$
p(s, x, t, y)=p(s+h, x+z, t+h, y+z) .
$$

The semigroup $P_{t} f(x)=\int_{\mathbb{R}^{d}} f(y) p_{t}(y-x) d y$ has $\Delta^{\alpha / 2}$ as infinitesimal generator ([1], [29], [3], [22]). Referring to the abstract, $p(s, x, t, y)$ is the funda- 
mental solution of $\partial_{t}-\Delta_{y}^{\alpha / 2}$ in the sense of distributions ([3]):

$$
\iint_{\mathbb{R} \mathbb{R}^{d}} p(s, x, t, y)\left[\partial_{t}+\Delta_{y}^{\alpha / 2}\right] \phi(t, y) d y d t=-\phi(s, x),
$$

where $s \in \mathbb{R}, x \in \mathbb{R}^{d}$, and $\phi \in C_{c}^{\infty}\left(\mathbb{R} \times \mathbb{R}^{d}\right)$. Here $C_{c}^{\infty}\left(\mathbb{R} \times \mathbb{R}^{d}\right)$ is the class of all infinitely differentiable compactly supported functions on $\mathbb{R} \times \mathbb{R}^{d}$, and

$$
\begin{aligned}
\Delta^{\alpha / 2} \varphi(y) & =\lim _{t \downarrow 0} \frac{P_{t} \varphi(y)-\varphi(y)}{t} \\
& =\frac{2^{\alpha} \Gamma((d+\alpha) / 2)}{\pi^{d / 2}|\Gamma(-\alpha / 2)|} \lim _{\varepsilon \downarrow 0} \int_{\{|z|>\varepsilon\}} \frac{\varphi(y+z)-\varphi(y)}{|z|^{d+\alpha}} d z, \quad y \in \mathbb{R}^{d} .
\end{aligned}
$$

A simple proof of (36) can be given by using Fourier transform in the space variable, and (33) (we omit the details).

We will assume that $q$ is relatively small for $p$. Let $(L \phi)(t, y)=\partial_{t} \phi(t, y)+$ $\Delta_{y}^{\alpha / 2} \phi(t, y)$. We also introduce $(Q \phi)(t, y)=q(t, y) \phi(t, y)$, the operation of multiplication by $q$. Referring to our previous notation we have $P^{q}=P Q$, and (28) now reads

$$
\widetilde{P}=\sum_{n=0}^{\infty}(P Q)^{n} P
$$

We can interpret (36) as

$$
P L \phi=-\phi \quad\left(\phi \in C_{c}^{\infty}\left(\mathbb{R} \times \mathbb{R}^{d}\right)\right) .
$$

This implies that

$$
\widetilde{P}(L+Q) \phi=-\phi \quad\left(\phi \in C_{c}^{\infty}\left(\mathbb{R} \times \mathbb{R}^{d}\right)\right) .
$$

Indeed, by (38),

$$
\widetilde{P}(L+Q) \phi=P L \phi+\sum_{n=1}^{\infty}(P Q)^{n} P L \phi+\sum_{n=0}^{\infty}(P Q)^{n+1} \phi=-\phi .
$$

The associativity of the operations involved, which we have used freely above, follows from Fubini's theorem. Indeed, $\phi \in C_{c}^{\infty}\left(\mathbb{R} \times \mathbb{R}^{d}\right)$ and $L \phi$ are bounded by a constant multiple of $p\left(\cdot, \cdot, t_{0}, y_{0}\right)$ for some $t_{0} \in \mathbb{R}, y_{0} \in \mathbb{R}^{d}$ (see the remarks on (8) in the proof of Theorem 2). This proves (39):

$$
\iint_{\mathbb{R}_{\mathbb{R}^{d}}} \widetilde{p}(s, x, t, y)\left[\partial_{t} \phi(t, y)+\Delta_{y}^{\alpha / 2} \phi(t, y)+q(t, y) \phi(t, y)\right] d y d t=-\phi(s, x),
$$

where $s \in \mathbb{R}, x \in \mathbb{R}^{d}$, and $\phi \in C_{c}^{\infty}\left(\mathbb{R} \times \mathbb{R}^{d}\right)$. In fact, $(s, x, t, y) \mapsto \widetilde{p}(s, x, t, y)$ is continuous, except when $s=t$. Indeed, continuity is first proved inductively for each $p_{n}$ in (10), by using an argument of uniform integrability. We omit the details of the proof, and refer the reader to a similar argument in the proof of [5, Lemma 14] (see also Lemma 9 and (19) above). The continuity 
of $\widetilde{p}=\widetilde{p}_{q}$ then follows from the locally (in time) uniform convergence of the series in (21).

Proof of Theorem 1. We consider the Gaussian transition density $g$ introduced in Section 1. This corresponds to $\alpha=2$ in (32), and an analogue of (36) holds for $g$ and the Laplacian with a similar proof. The above discussion of the fractional Laplacian also applies to the Laplacian, provided $q$ is relatively small with respect to $g$.

Apart from obvious similarities, there exist important differences between $p(0<\alpha<2)$ and $g(\alpha=2)$. For instance the global decay of $p$ in space is qualitatively different from that of $g$. In fact, we have the following estimate of $p$ (cf. (34) and see, e.g., [6] for a proof).

Lemma 10. There exists $c=c(d, \alpha)$ such that, for all $z \in \mathbb{R}^{d}$ and $t>0$,

$$
c^{-1}\left(\frac{t}{|z|^{d+\alpha}} \wedge t^{-d / \alpha}\right) \leq p_{t}(z) \leq c\left(\frac{t}{|z|^{d+\alpha}} \wedge t^{-d / \alpha}\right) .
$$

This power-type asymptotics yields the following 3P Theorem ([5]).

TheOREM 4. There exists a constant $c=c(d, \alpha)$ such that

$$
p(s, x, u, z) \wedge p(u, z, t, y) \leq c p(s, x, t, y) \quad \text { for } x, z, y \in X, s, u, t \in \mathbb{R} .
$$

For numbers $a, b \geq 0$ we have $a b=(a \vee b)(a \wedge b)$ and $a \vee b \leq a+b$. Therefore Theorem 4 yields the following variant form with five occurrences of $p$ :

$$
p(s, x, u, z) p(u, z, t, y) \leq c p(s, x, t, y)[p(s, x, u, z)+p(u, z, t, y)] .
$$

From this and Lemma 9 we immediately obtain the following consequence.

Corollary 11. For the transition density of the fractional Laplacian $\Delta^{\alpha / 2}$ with $0<\alpha<2$, the parabolic Kato class equals the relative Kato class.

For instance, if $\alpha<d$, then Lemma 10 (see also (35)) yields

$$
\int_{0}^{h} p_{t}(z) d t \approx|z|^{\alpha-d} \wedge\left[h^{2}|z|^{-d-\alpha}\right], \quad z \in \mathbb{R}^{d}, h>0 .
$$

If $0<\varepsilon \leq \alpha,|q(u, z)| \leq|z|^{-\alpha+\varepsilon}, s \in \mathbb{R}, x \in \mathbb{R}^{d}$, and we let $h \rightarrow 0$, then

$$
\int_{s}^{s+h} \int_{\mathbb{R}^{d}} p(s, x, u, z)|q(u, z)| d z d u \leq \int_{\mathbb{R}^{d}}|z|^{-\alpha+\varepsilon} \int_{0}^{h} p_{t}(z) d t d z \rightarrow 0 .
$$

Therefore each such $q$ belongs to the parabolic Kato class, and so it is relatively Kato. Let us note that the local (in time) comparability of the Schrödinger semigroups of the fractional Laplacian for $q$ in the (parabolic) Kato class is a new result even in the autonomous case of [3]. We also refer the reader to [31] for recent Gaussian results, with a definition [31, (1.2)] not 
unrelated to our relative Kato condition (see also [10, Definition 3.1] and [16, Lemma 5.2], [27, Theorem 3.10]).

For a study of other consequences of the Kato condition for autonomous additive perturbations we refer the reader to [32], [4], [5].

6. Further discussion. For the sake of clarity, let us add a comment on the lower bound in (27). If $q$ is relatively Kato for $g$ (see Section 1) then for $\widetilde{g}=\widetilde{g}_{q}$ we have

$$
\frac{g_{q}(s, x, t, y)}{g(s, x, t, y)} \geq \exp \left(-\int_{s}^{t} \int_{X} \frac{g(s, x, u, z) g(u, z, t, y)}{g(s, x, t, y)} q_{-}(u, z) d z d u\right) .
$$

An analogous estimate holds for the transition density $p$ of the fractional Laplacian $\Delta^{\alpha / 2}$. These results follow from the fact that

$$
R_{\lambda}:=\left(I+\lambda P^{q_{-}}\right)^{-1} P^{q_{-}}, \quad \lambda>0,
$$

is a sub-Markov resolvent (of $P^{q_{-}}$), a unique kernel satisfying

$$
R_{\lambda}+\lambda P^{q_{-}} R_{\lambda}=P^{q_{-}} .
$$

For further background, we refer the reader to [2, 7.2-7.7]. By an argument of log-convexity (see, e.g., [20] or [18, p. 429], and also [2, 8.1-8.2]), we obtain (41). It is noteworthy that one only needs relative boundedness of $q_{-}$to obtain satisfactory lower bounds for $\widetilde{p}$, as defined by (8).

We omit the (standard) details for two reasons. Firstly, our emphasis in this paper is on upper bounds, or non-explosion results. Secondly, in view of possible generalizations mentioned below it seems economical to postpone the full discussion to a future paper. We remark that, in principle, the lower bound (41) is well known (see, e.g., [11], [3]).

We now comment on possible and forthcoming generalizations of our results. It is possible to extend the present results to more general integral kernels or to measures (rather than functions $q$; see [20], [26], [16] for a related study). In fact, considering $q(d u d z)=\eta \delta_{u_{0}}(d u) d z$, where $\eta \geq 1$ and $\delta_{u_{0}}$ is the probability measure concentrated at $u_{0}$, shows that $\widetilde{p}_{q}$ may explode in finite time $u_{0}$.

The technique based on Theorem 3 applies to more general additive perturbations (of the generator). In studying these, one should attempt a natural and general description (in terms of $p$ ) of a class of perturbations which lead to comparability theorems. In this connection we refer to [25] for a discussion of nonlocal perturbations of the fractional Laplacian, and to [5] for a study of gradient perturbations of $\Delta^{\alpha / 2}$ under the assumption that $1<\alpha<2$.

There is a deep well-known connection of Schrödinger operators to the theory of multiplicative functionals of Markov processes (see, e.g., [16]). We now discuss this connection in the case of the Wiener process $Y$ in $\mathbb{R}^{d}$, 
defined by the transition density $g$ of Section 1 (note that $Y_{t}=B_{2 t}$, where $B$ is the standard Brownian motion). Let $\mathbb{E}_{s, x}$ and $\mathbb{P}_{s, x}$ be respectively the expectation and distribution of the process starting at the point $x \in \mathbb{R}^{d}$ at time $s \in \mathbb{R}$, so that $\mathbb{P}_{s, x}\left[Y_{t} \in A\right]=\int_{A} g(s, x, t, y) d y$, where $Y_{t}$ is the canonical continuous coordinate process evaluated at time $t>s$. For $y \in \mathbb{R}^{d}$ we let $\mathbb{E}_{s, x}^{t, y}$ and $\mathbb{P}_{s, x}^{t, y}$ denote, respectively, the expectation and distribution of the process starting at $x$ at time $s$ and conditioned to reach $y$ at time $t$ (Brownian bridge). The process is defined by the transition probability density function $r\left(u_{1}, z_{1}, u_{2}, z_{2}\right)=g\left(u_{1}, z_{1}, u_{2}, z_{2}\right) g\left(u_{2}, z_{2}, t, y\right) / g\left(u_{1}, z_{1}, t, y\right)$, where $s \leq u_{1}<$ $u_{2}<t$ and $z_{1}, z_{2} \in \mathbb{R}^{d}$. Thus, the finite-dimensional distributions are given by the density functions

$$
\frac{g\left(s, x, u_{1}, z_{1}\right) g\left(u_{1}, z_{1}, u_{2}, z_{2}\right) \ldots g\left(u_{n}, z_{n}, t, y\right)}{g(s, x, t, y)},
$$

and we have the following disintegration of $\mathbb{P}_{s, x}$ :

$$
\begin{aligned}
\mathbb{P}_{s, x}\left(Y_{u_{1}} \in A_{1}, \ldots, Y_{u_{n}} \in A_{n} ; Y_{t} \in B\right) \\
\quad=\int_{B} \mathbb{P}_{s, x}^{t, y}\left(Y_{u_{1}} \in A_{1}, \ldots, Y_{u_{n}} \in A_{n}\right) g(s, x, t, y) d y .
\end{aligned}
$$

Here $x, z_{1}, \ldots, z_{n}, y \in \mathbb{R}^{d}, s \leq u_{1}<\cdots<u_{n}<t$, and $A_{1}, \ldots, A_{n} \subset$ $\mathbb{R}^{d}$ are Borel sets. Consider the multiplicative functional $([11]) e_{q}(s, t)=$ $\exp \left(\int_{s}^{t} q\left(u, Y_{u}\right) d u\right)$. We have

$$
\mathbb{E}_{s, x}^{t, y} e_{q}(s, t)=\sum_{n=0}^{\infty} \frac{1}{n !} \mathbb{E}_{s, x}^{t, y}\left(\int_{s}^{t} q\left(u, Y_{u}\right) d u\right)^{n} .
$$

According to (9) and (42),

$$
\begin{aligned}
\mathbb{E}_{s, x}^{t, y} \int_{s}^{t} q\left(u, Y_{u}\right) d u & =\int_{s}^{t} \int_{\mathbb{R}^{d}} \frac{g(s, x, u, z) q(u, z) g(u, z, t, y)}{g(s, x, t, y)} d z d u \\
& =\frac{g_{1}(s, x, t, y)}{g(s, x, t, y)} .
\end{aligned}
$$

Furthermore,

$$
\begin{aligned}
\mathbb{E}_{s, x}^{t, y} & \frac{1}{2}\left(\int_{s}^{t} q\left(u, Y_{u}\right) d u\right)^{2}=\mathbb{E}_{s, x}^{t, y} \int_{s u}^{t} \int_{u}^{t} q\left(u, Y_{u}\right) q\left(v, Y_{v}\right) d v d u \\
\quad & =\int_{s u}^{t} \int_{\mathbb{R}^{d}} \int_{\mathbb{R}^{d}} \frac{g(s, x, u, z) g(u, z, v, w) g(v, w, t, y)}{g(s, x, t, y)} q(u, z) q(v, w) d w d z d v d u \\
& =\int_{s}^{t} \int_{\mathbb{R}^{d}} \frac{g(s, x, u, z) g_{1}(u, z, t, y)}{g(s, x, t, y)} q(u, z) d z d u=\frac{g_{2}(s, x, t, y)}{g(s, x, t, y)} .
\end{aligned}
$$


By induction, for every $n=0,1, \ldots$,

$$
\frac{1}{n !} \mathbb{E}_{s, x}^{t, y}\left(\int_{s}^{t} q\left(u, Y_{u}\right) d u\right)^{n}=\frac{g_{n}(s, x, t, y)}{g(s, x, t, y)}
$$

hence

$$
\mathbb{E}_{s, x}^{t, y} e_{q}(s, t)=\frac{\widetilde{g}_{q}(s, x, t, y)}{g(s, x, t, y)} .
$$

We may interpret $\widetilde{g}_{q}(s, x, t, y) / g(s, x, t, y)$ as the eventual inflation of mass of the Brownian particle moving from $(s, x)$ to $(t, y)$. The mass grows multiplicatively where $q>0$, and decreases where $q<0$. Thus we may consider the results of this paper as uniform bounds for this mass.

The following example illustrates inequality (20). For a general transition density $p$ we consider a function $q(u, z)=q(u)$, depending only on time and locally integrable in time. It easily follows from (9) that

$$
\frac{\widetilde{p}_{q}(s, x, t, y)}{p(s, x, t, y)}=\exp \left(\int_{s}^{t} q(u) d u\right) .
$$

Note that $\eta^{*}(|q|)=0$ in this example, whilst the emphasis in Theorem 3 is on $\eta^{*}(|q|)>0$.

In view of (44) and (7), the relative Kato class may be considered as a Kato class for the conditional processes (bridges); see (29, 30).

Acknowledgments. We thank Panki Kim and the referees of the paper for discussion, corrections and insightful suggestions. The results of this paper were presented at the 2nd International Conference on Stochastic Analysis and Its Applications, May 28-31, 2008 at Seoul National University.

\section{References}

[1] C. Berg and G. Forst, Potential Theory on Locally Compact Abelian Groups, Springer, New York, 1975.

[2] J. Bliedtner and W. Hansen, Potential Theory, Universitext, Springer, Berlin, 1986.

[3] K. Bogdan and T. Byczkowski, Potential theory for the $\alpha$-stable Schrödinger operator on bounded Lipschitz domains, Studia Math. 133 (1999), 53-92.

[4] - - - Potential theory of Schrödinger operator based on fractional Laplacian, Probab. Math. Statist. 20 (2000), 293-335.

[5] K. Bogdan and T. Jakubowski, Estimates of heat kernel of fractional Laplacian perturbed by gradient operators, Comm. Math. Phys. 271 (2007), 179-198.

[6] K. Bogdan, A. Stós, and P. Sztonyk, Harnack inequality for stable processes on d-sets, Studia Math. 158 (2003), 163-198.

[7] K. Bogdan and P. Sztonyk, Harnack's inequality for stable Lévy processes, Potential Anal. 22 (2005), 133-150.

[8] - - - Estimates of the potential kernel and Harnack's inequality for the anisotropic fractional Laplacian, Studia Math. 181 (2007), 101-123. 
[9] Z.-Q. Chen and R. Song, Intrinsic ultracontractivity and conditional gauge for symmetric stable processes, J. Funct. Anal. 150 (1997), 204-239.

[10] - - - General gauge and conditional gauge theorems, Ann. Probab. 30 (2002), $1313-1339$.

[11] K. L. Chung and Z. X. Zhao, From Brownian Motion to Schrödinger's Equation, Grundlehren Math. Wiss. 312, Springer, Berlin, 1995.

[12] M. Cranston, E. Fabes, and Z. Zhao, Conditional gauge and potential theory for the Schrödinger operator, Trans. Amer. Math. Soc. 307 (1988), 171-194.

[13] E. B. Davies, One-Parameter Semigroups, London Math. Soc. Monogr. 15, Academic Press, London, 1980.

[14] N. Dunford and J. T. Schwartz, Linear Operators. I. General Theory, Pure Appl. Math. 7, Interscience Publ., New York, 1958.

[15] R. L. Graham, D. E. Knuth, and O. Patashnik, Concrete Mathematics, AddisonWesley, Reading, MA, 1994.

[16] A. Gulisashvili, Classes of time-dependent measures, non-homogeneous Markov processes, and Feynman-Kac propagators, Trans. Amer. Math. Soc. 360 (2008), 40634098.

[17] A. Gulisashvili and J. A. van Casteren, Non-autonomous Kato classes and Feynman-Kac propagators, World Sci., Hackensack, NJ, 2006.

[18] W. Hansen, Harnack inequalities for Schrödinger operators, Ann. Scuola Norm. Sup. Pisa Cl. Sci. (4) 28 (1999), 413-470.

[19] —, Uniform boundary Harnack principle and generalized triangle property, J. Funct. Anal. 226 (2005), 452-484.

[20] -, Global comparison of perturbed Green functions, Math. Ann. 334 (2006), 643678.

[21] —, Simple counterexamples to the 3G-inequality, Expo. Math. 24 (2006), 97-102.

[22] N. Jacob, Pseudo Differential Operators and Markov Processes. Vol. I, Imperial College Press, London, 2001.

[23] T. Jakubowski, The estimates for the Green function in Lipschitz domains for the symmetric stable processes, Probab. Math. Statist. 22 (2002), 419-441.

[24] T. Kato, Perturbation Theory for Linear Operators, Classics Math., Springer, Berlin, 1995 (reprint of the 1980 edition).

[25] P. Kim and Y.-R. Lee, Generalized 3G theorem and application to relativistic stable process on non-smooth open sets, J. Funct. Anal. 246 (2007), 113-143.

[26] P. Kim and R. Song, Estimates on Green functions and Schrödinger-type equations for non-symmetric diffusions with measure-valued drifts, J. Math. Anal. Appl. 332 (2007), 57-80.

[27] V. Liskevich, H. Vogt, and J. Voigt, Gaussian bounds for propagators perturbed by potentials, J. Funct. Anal. 238 (2006), 245-277.

[28] D. Revuz, Markov Chains, 2nd ed., North-Holland Math. Library 11, North-Holland, Amsterdam, 1984.

[29] K. Yosida, Functional Analysis, Classics Math., Springer, Berlin, 1995.

[30] Q. S. Zhang, On a parabolic equation with a singular lower order term. II. The Gaussian bounds, Indiana Univ. Math. J. 46 (1997), 989-1020.

[31] —, A sharp comparison result concerning Schrödinger heat kernels, Bull. London Math. Soc. 35 (2003), 461-472. 
[32] Z. Zhao, A probabilistic principle and generalized Schrödinger perturbation, J. Funct. Anal. 101 (1991), 162-176.

Institute of Mathematics and Computer Science

Fakultät für Mathematik

Wrocław University of Technology

Universität Bielefeld

Wybrzeże Wyspiańskiego 27

50-370 Wrocław, Poland

E-mail: Krzysztof.Bogdan@pwr.wroc.pl Postfach 100131

Tomasz.Jakubowski@pwr.wroc.pl

Received September 4, 2007

Revised version September 22, 2008 